\title{
CARACTERÍSTICAS COMPETENCIALES DEL PSICOPEDAGOGO: UNA APROXIMACIÓN DESDE LA COMUNIDAD AUTÓNOMA DE GALICIA
}

\author{
THE PSYCHOPEDAGOGUE'S PROFILE OF COMPETENCIES: \\ AN APPROACH FROM THE AUTONOMOUS COMMUNITY OF GALICIA \\ Carmen Delia García-Fuentes de la Fuente*, Olga Rodríguez Rodríguez** \\ Universidade da Coruña \\ Sonia Rodríguez Fernández*** \\ Universidad de Granada
}

\section{RESUMEN}

En este trabajo se presenta una revisión sobre aspectos teóricos del ámbito de las competencias profesionales y una reflexión sobre las características competenciales del/la psicopedagogo/a. Se concluye el trabajo con una aplicación práctica en una muestra de la Comunidad Autónoma de Galicia.

Palabras clave: competencias profesionales, psicopedagogo, características competenciales.

\begin{abstract}
This article shows a revision about the theoretical aspects in the area of professional competencies and a discussion about the competence features of the psycho-pedagogue. The work ends with a practical application in a sample of the Galician Autonomous Comunity.
\end{abstract}

Key vords: profesional abilities, psicopedagogue, competence features

* Carmen Delia García-Fuentes de la Fuente: cdelia@udc.es es Profesora Titular de la Universidade Da Coruña, área MIDE, en el Departamento de Filosofía y Métodos de Investigación en Educación. Línea de trabajo: Diagnóstico de los estilos de aprendizaje en el contexto educativo y de las organizaciones, mujer y familia.

** Olga Rodríguez Rodríguez por la Universidade. Lda. En Psicopedagogía, D.E.A. y MBA en Marketing y Dirección Comercial por la Universidade Da Coruña, formadora en el ámbito mujer en el Proyecto Inserta de la U.E.

*** Sonia Rodríguez Fernández: soniarf@ugr.es es Profesora Asociada y Dra. por la Universidad de Granada Línea de trabajo: Orientación y evaluación de necesidades en Educación Superior. 


\section{Introducción}

La perspectiva educativa actual presenta una serie de limitaciones de carácter general, algunas de ellas pueden ser imputadas a los cambios que se producen en la sociedad a lo largo de las últimas décadas.

Diversos autores coinciden en señalar características de la sociedad que están presentes y condicionan aspectos interrelacionados. En este sentido Echeverría (1999) Carneiro (1999) y Ferrer (2001) entre otros muchos, mencionan los rasgos más relevantes que a su modo de ver, guardan relación con los cambios educativos del momento y estos serían:

- Una sociedad interdependiente, que traspasa las fronteras de los países cuestión que produce una deslocalización de las empresas, movimientos migratorios, pérdida del control político y crecimiento del factor multicultural.

- Una sociedad envejecida por disminución de la población perteneciente a la franja de edad de los más jóvenes y un incremento del sector de los mayores.

Lógicamente todo esto acarrea consecuencias de tipo económico, sanitario laboral y social.

Además nos encontramos con una sociedad con exceso de información que contrasta con aquellos reductos sociales que constituyen la bolsa de la exclusión social en este caso por falta de información.

Una sociedad del ocio, con disminución de horas de trabajo y aumento de actividades lúdicas.

Una sociedad doméstica con dos fenómenos antagónicos, una vuelta al hogar y una crisis familiar por la abundancia de familias rotas y desestructuradas.

Una sociedad urbana de tal forma que, según la ONU, a mediados del 2025 más de las dos terceras partes de la sociedad será urbana aunque esta sociedad estará tutorizada por un entorno virtual que hará de aglutinante entre los elementos humanos, económicos, organizativos y estructurales de las nuevas ciudades.

Con esta perspectiva, es lógico que el profesional de la psicopedagogía debe asumir, una serie de responsabilidades que le sitúen como pieza significativa en el ámbito escolar y social. Esto no sucede en la actualidad, los psicopedagogos se esfuerzan en encontrar su lugar en todos estos ámbitos y resulta difícil vertebrar las competencias básicas que deben presentar, para desarrollar de la manera más adecuada posible su función.

\section{La evolución del concepto de competencia}

El concepto de competencia surge de la necesidad de valorar no sólo el conjunto de los conocimientos apropiados (saber) y las habilidades y destrezas (saber hacer) desarrolladas por una persona, sino de apreciar su capacidad de emplearlas para responder a situaciones, resolver problemas y desenvolverse en el mundo. Igualmente, implica una mirada a las condiciones del individuo y disposiciones con las que actúa, es decir, al componente actitudinal y valorativo (saber ser) que incide sobre los resultados de la acción.

Las competencias, fundamentalmente, son las respuestas profesionales que una persona da a los requerimientos de su puesto de trabajo. La síntesis de las definiciones de compe- 
tencias efectuada por Pérez Escoda (2001) aporta aquellos elementos más destacables del concepto:

- El concepto es aplicable a las personas (individualmente o en forma grupal).

- Implica unos conocimientos "saberes", unas habilidades "saber hacer", y unas actitudes y conductas "saber estar" integrados entre sí.

- Incluye las capacidades y procedimientos informales además de las formales.

- Es indisociable de la noción de desarrollo y de aprendizaje continuo unido a la experiencia.

- Constituye un capital o potencial de actuación vinculado a la capacidad de movilizarse o ponerse en acción.

- Se inscribe en un contexto determinado que posee unos referentes de eficacia y que cuestiona su transferibilidad.

Las competencias han ido evolucionando en su meta de dar respuesta a estos diversos requerimientos. Tal como señala Echeverría (2002), las primeras definiciones de competencias aludían a la capacidad de la persona para el desempeño de las actividades o funciones de su puesto de trabajo. Lo que dio lugar al enfoque de competencias centrado en la tarea.

En un segundo momento, se prestó atención a los rasgos y características del profesional excelente, aquel capaz de dar el máximo resultado en su actividad profesional, aquellas competencias que diferenciaban entre el profesional que cumplía su tarea y el profesional que destacaba en dicho logro. En este nuevo enfoque centrado en el perfil no sólo se prestaba atención a la base técnica y profesional sino que se empezó a indagar acerca de las competencias clave, aquellas competencias dotaban de flexibilidad, capacidad de aprendizaje y superación, y todos aquellos rasgos que se estimaran necesarios para llegar a ser este tipo de profesional excelente.

Finalmente, se ha llegado a conceptualizar un enfoque de competencia de naturaliza holística y compleja, cuyas diversas conceptualizaciones intentan recoger y abordar tanto los elementos de tarea, de excelencia profesional como de desarrollo y adaptación al entorno complejo y global en que se desarrolla la actividad profesional.

En esta concepción de la competencia se sitúa lo indicado por Echeverría (2005) cundo identifica las competencias como la puesta en práctica del conjunto de conocimientos, procedimientos, actitudes y capacidades con las que una persona cuenta. Dichas capacidades son necesarias para enfrentarse de forma efectiva a las actividades de una profesión y de un puesto de trabajo con el nivel y la calidad exigida. Al tiempo que se resuelven autónomamente y de forma creativa los problemas emergentes y se colabora en la organización del trabajo y en la creación de un buen clima sociolaboral.

Tal como se puede observar, en esta consideración de las competencias inciden diversos aspectos de tipo profesional y de tipo personal.

\section{La dimensión profesional-personal del psicopedagogo}

Es preciso entender el profesional de la psicopedagogía como un integrante más dentro del mundo del trabajo aunque con unas connotaciones singularizadoras que vienen impues- 
tas por su posible vinculación a varios ámbitos desde la educación formal y también desde la educación no formal.

Del Rincón (2001) sitúa al psicopedagogo en las grandes áreas de la orientación en educación primaria y secundaria, el psicopedagogo en los equipos específicos de orientación, en la universidad, el psicopedagogo como profesional de la acción social y el psicopedagogo en el ámbito de las organizaciones, generalmente inmerso en el área de recursos humanos.

Las capacidades profesionales son objeto de estudio en las últimas décadas, en ellas se incluyen, según indica Sanz Oro (1998), todo tipo de saberes, actitudes y destrezas cuya finalidad es realizar actividades conexionadas con una profesión.

Tal como señala Echevarría (2001), en épocas anteriores primaba la función del profesional integrado en la empresa, se enfatizaba la posesión de capacidades para desarrollar una determinada profesión, se mantenía una dinámica de interdependencia de acción entre funciones, con objeto de mejorar el sistema de la organización. A partir de los años ochenta se habla de habilidades y destrezas para ejercer una amplia gama de actividades laborales, así se emplea el término cualificación. Este termino es definido por varios autores, entre ellos Blovin y Bergeron (1997) que la identifican como el conjunto de conocimientos, de aptitudes y de experiencia adquirida para ejercer un empleo.

Sanz y Sobrado (1998) dan un paso más y definen la competencia profesional del orientador como el conjunto de actitudes saberes y habilidades aprendidas mediante la formación o la experiencia laboral que posibilita realizar y desempeñar roles y situaciones de trabajo requeridos en la profesión del orientador.

Continuando con lo señalado por Echevarría (2001) nos encontramos que el puesto de trabajo o el ejercicio de la profesión comportan capacidades, conocimientos y expectativas de conocimiento para realizar la tarea asignada. Para este autor, cualquier profesional debe poseer la competencia técnica, es decir saber, la competencia participativa, saber estar, la competencia metodológica saber hacer y la competencia personal, saber ser.

La declaración de Bolonia (1989) y el comunicado de Praga (2001) tal como señalan González y Wagenaar (2003) entre otras muchas cuestiones, pretenden coordinar los objetivos que fija la comunidad académica para la Educación Superior con los perfiles académicos y profesionales que exige la sociedad.

Algunos de los aspectos resultantes de las aspiraciones de la Educación Superior en Europa se ha puesto de manifiesto en el proyecto Tuning (2001-2002) cuyo objetivo es buscar puntos de acuerdo de convergencia y entendimiento mutuo, protegiendo la diversidad. En este proyecto se ha adoptado una metodología en la que se han introducido algunos aspectos, lógicamente, uno de ellos es, la consideración de los resultados del aprendizaje. A esos resultados de aprendizaje se le identifica con el conjunto de competencias, es decir conocimientos, comprensión y habilidades que se espera que el estudiante domine, comprenda y demuestre después de completar un proceso corto o largo de aprendizaje.

Esto tiene su correlato en cada una de las titulaciones que se tratan en este proyecto Tuning entre las que se encuentra la de Ciencias de la Educación.

Paralelamente existen indicaciones dadas por los teóricos de la educación referentes a lo que debe aportar el psicopedagogo en el ámbito escolar y también existen indicaciones, aunque menos, sobre la misión a desarrollar por los mismos, en el ámbito de las organiza- 
ciones, tanto públicas como privadas. En este sentido García Nieto (1999) destaca las siguientes:

- Ayudar a comprender las bases ideológicas, psicológicas y sociológicas sobre las que se apoya el currículo escolar.

- Ayudar a comprender los términos específicos novedosos para los miembros de la comunidad educativa.

- Analizar con los demás docentes lo que se pretende conseguir con los planteamientos educativos actuales.

- Apoyo en la cualificación y en la formación del profesorado.

- Coordinar a los realizadores de la acción tutorial y los proyectos educativos de los Centros.

- Asesorar a los órganos directivos de aspectos técnicos así como a los componentes del entorno familiar y el contexto ambiental.

- Atención a la diversidad.

A lo anteriormente indicado sería conveniente añadir todo lo relacionado con la acción del psicopedagogo en el ámbito de la educación no formal, asesoramiento para la formación y el trabajo, asesoramiento en las tecnologías de la información TIC, ocio, tiempo libre y orientación de los mayores.

Por otra parte es conveniente hacer notar la incidencia de la orientación en la Educación Superior, en la investigación y en la evaluación. El ámbito personal y el profesional se presentan particularmente relacionados en profesiones como es la del psicopedagogo.

También García Nieto (2000) recopila algunas de las caracterizaciones especialmente adecuadas para perfilar la línea personal de actuación del psicopedagogo, destacamos entre ellas las siguientes:

- Sentido de responsabilidad profesional, con la consciencia de que el buen o mal hacer de este profesional puede influir de forma decisiva en las personas que acuden a su consejo y ayuda.

- Competencia profesional, preparación científica rigurosa y técnica actualizada y renovada.

- Utilización prudente y confidencial de la información, ya que en el psicopedagogo confluyen informaciones sobre problemas y tensiones familiares así como conflictos profesionales entre distintos niveles, la intimidad de las personas solo les pertenece a ellos y no debe vulnerarse nada más que por causas mayores y con consentimiento de los interesados.

- Relaciones interpersonales adecuadas, evitando crear dependencias personales inadecuadas, hipotecando sentimientos en función de otros intereses.

- Respeto a las personas, ni la autoridad ni la sabiduría deben legitimar el trato impropio hacia las personas, por encima de los problemas está la dignidad de las mismas.

- Madurez y equilibrio personales, Las manifestaciones del psicopedagogo pueden marcar pautas de comportamiento y llevar a toma de decisiones irreversible, el sentido ético, el rigor profesional y la integridad personal serán las realizaciones óptimas para el psicopedagogo. 
- Sensibilidad hacia problemas de los otros, esto supone saber acoger, saber escuchar y saber comprender los problemas por encima de cualquier recompensa o prestigio social o profesional.

Abundando en más opiniones, tal como indicó García-Fuentes (2002), el psicopedagogo ejerce una profesión que se enclava entre las profesiones de ayuda, por tanto, lo hacen especialmente eficaz algunas de las siguientes notas caracterizadoras:

- Una buena relación interpersonal que hace su profesionalidad más efectiva.

- Dar un alto valor al tema de la confidencialidad.

- Deseo y puesta en práctica de técnicas de ayuda al semejante.

- Utilización de técnicas comunes del tipo de escucha, acogida, relación empática, entrevista y muchas otras.

Por su parte Bisquerra (2004) enfatiza la importancia de la autonomía personal y la autogestión personal y relaciona con ella otras características que reseñamos sintéticamente:

- Autoestima, imagen positiva de uno mismo y mantener buenas relaciones con uno mismo.

- Automotivación o capacidad para implicarse en actividades diversas del ámbito personal, social, cultural.

- Actitud positiva, ser constructivo para uno mismo y para la sociedad, intención de ser bueno, justo, caritativo y compasivo.

- Responsabilidad, intención de implicarse en comportamientos seguros, saludables y éticos, asumiendo responsabilidad en la toma de decisiones.

- Análisis crítico de las normas sociales, capacidad para evaluar críticamente los mensajes sociales, culturales y de los mass media, relativos a normas sociales y comportamientos personales.

- Buscar ayuda y recursos, capacidad para buscar apoyo y asistencia y saber acceder a los recursos disponibles apropiados.

- Autoeficacia emocional, la persona se siente como se quiere sentir, aceptando su propia experiencia emocional tanto si es única y excéntrica como si es convencional, mostrándose en consonancia con los propios valores morales.

De lo anteriormente explicitado se extraen abundantes conclusiones tendentes al fortalecimiento de la figura del psicopedagogo en cuanto su perfeccionamiento personal-profesional. Efectivamente las indicaciones referidas sobre autoestima, automotivación, y actitud positiva generan un crecimiento ascendente que repercute y da como resultado la autoeficacia en el ejercicio de la profesión.

\section{Competencias del psicopedagogo}

Existe un estudio de investigación de Repetto, Malik, Ferrer, Manzano y Hiebert (2003) en él que se ha recabado información de diversos países y que fue hecho público en Berna, durante la celebración del Congrés International de l'AIOSP. Las competencias resultantes del análisis de los datos recogidos, han sido adoptadas por la asamblea general de la AIOSP, 
tal como figura en la publicación de la conferencia en Quality Development in Vocational Counselling and Training.

En el citado estudio, se presenta una diferenciación entre competencias fundamentales y competencias especializadas o específicas. Entre las primeras, se incluyen las siguientes:

1. Demostrar comportamiento ético y actitud profesional adecuada en el cumplimiento de los roles y responsabilidades.

2. Demostrar mediación e iniciativa para anticipar el aprendizaje, desarrollo profesional y cuestiones personales de los clientes.

3. Demostrar conocimiento y valoración de las diferentes culturas de los clientes para interaccionar de forma efectiva con la población en general.

4. Integrar teoría e investigación en la práctica de la orientación, desarrollo profesional, consejo y consulta.

5. Capacidad para diseñar, implementar y evaluar programas de orientación y consejo y las intervenciones correspondientes.

6. Demostrar consciencia de las propias capacidades y limitaciones.

7. Capacidad de comunicación con los colegas o con los clientes utilizando el lenguaje apropiado.

8. Conocimiento y puesta al día de las tendencias educacionales, de formación, empleo, marcha del trabajo y cuestiones sociales.

9. Sensibilidad para implicarse en cuestiones sociales y multiculturales.

10. Capacidad de cooperación efectiva en un equipo de profesionales.

11. Demostrar el conocimiento del proceso de desarrollo de la carrera a lo largo de toda la vida.

En el apartado de competencias específicas se distinguen diez áreas especializadas: diagnóstico, orientación educativa, desarrollo de la carrera, counseling, información, consulta, investigación, gestión de programas y servicios, desarrollo comunitario, y por último, empleo.

Como fácilmente puede deducirse es extenso el listado de competencias que resultan de este primer estudio caracterizador, esta iniciativa ha suscitado un amplio interés en los orientadores de los países implicados, esto es susceptible de ser completado y perfilado en el devenir de la labor orientadora e investigadora.

También existe un campo a considerar, la ética profesional, para la que el psicopedagogo debe tener una especial sensibilidad.

La Asociación Internacional de Orientación Escolar y Profesional AIOSP publica en 1995 una nueva relación de normas éticas que incluyen los principios éticos que subyacen a toda labor orientadora y sobre todo el código al que se adhieren los miembros que componen dicha asociación internacional. Estas normas están divididas en cuatro grandes apartados.

El primero, sobre responsabilidades éticas con los clientes, es decir, los sujetos pacientes de la orientación contienen diez postulados explicativos de los cuales reseñamos solamente la temática: 
I.1. Sobre el respeto a la dignidad de cada persona.

I.2. Sobre la obligatoriedad de ejercer la orientación hacia las personas sin discriminación alguna.

I.3. Versa sobre la posibilidad de orientación en el ámbito escolar, profesional, personal y social, remitiendo a los clientes a otros especialistas cuando el orientador por sí mismo un pueda satisfacer las necesidades requeridas.

I.4. Sobre la posibilidad de informar a los clientes de los objetivos y técnicas de orientación, reglas deontológicas guardando la confidencialidad.

I.5. Sobre la obligatoriedad de evitar imponer a los clientes, valores, modos de vida, etc.

I.6. Explicarán el resultado de los tests de forma clara y con lenguaje adecuado para la comprensión.

I.7. Comunicarán a los clientes los mejores avances en nuevas tecnologías para que se aseguren la adaptación y el acceso al empleo.

I.8. Se guardarán de manifestar sus competencias profesionales sin falsedades ni exageraciones.

I.9. Evitarán los conflictos de intereses que puedan ser nocivos para los clientes.

I.10. Dirigirán a los clientes a los servicios apropiados cuando su ayuda no resulte la más solicitada y adecuada

El segundo sobre actitudes hacia colegas y asociados profesionales, aquí se incluyen cinco postulados.

II.1. Sobre las buenas relaciones con otros colegas.

II.2. Informarán a los colegas sobre ciertos trabajos de orientación escolar y profesional.

II.3. Informarán a los directores sobre de los datos exactos sobre necesidades de orientación.

II.4. Colaborarán con los colegas en la observación del código deontológico.

II.5. En casa de divergencia entre el código deontológico y los directivos o en caso de no cooperación, buscarán los medios adecuados para poder remediar la situación conflictiva.

El tercero versa sobre los organismos gubernamentales y los comunitarios, y el cuarto sobre las responsabilidades respecto a la investigación y a los procesos relacionados con ella, que incluye dos postulados:

IV.1. Sobre la conveniencia de poseer las competencias.

IV.2. Sobre la contribución a la mejora de la orientación.

El quinto incluye las responsabilidades del orientador a nivel individual que comprende siete postulados. En este apartado se incluye de nuevo las competencias para ejercer la profesión, la adhesión a los principios humanistas, la supervisión de sus conocimientos, del compromiso de los valores y finalmente de la conveniencia de informarse de forma confidencial cuando las cuestiones deontológicas resulten imprecisas o ambiguas en el ejercicio 
de la profesión comunicándose directamente con la secretaría general de la AIOSP para obtener el esclarecimiento.

\section{Una aplicación práctica en la Comunidad Autónoma de Galicia}

A continuación presentamos una aplicación práctica a modo de estudio piloto sobre las características más destacadas. Responde a una idea de aspiración y no de realidad evidenciada en el momento actual ya que, la mayoría de los encuestados presentan una idea del psicopedagogo influenciada por el ejercicio profesional en niveles de diplomatura.

La población de la que se extrae la muestra la configuran alumnos y alumnas de la Licenciatura de Psicopedagogía del último año de la Universidad de A Coruña. La muestra esta compuesta de 44 sujetos, la mayoría mujeres (93\%), con una edad media de 22 años, que han cursado otros estudios relacionados con la educación, destacando las especialidades de Educación Infantil (43\%) y Educación Primaria (39\%).

Como objetivo prioritario perseguimos el hacer reflexionar a los futuros pedagogos sobre aquellas competencias que deberían alcanzar para ejercer, con mayor eficacia, su futuro profesional.

El instrumento utilizado ha sido un cuestionario (anexo I) que forma parte del Proyecto Tuning, el cual ha sido distribuido a los alumnos en el horario de clase. Dicho cuestionario está formado por tres partes. La primera parte contiene una serie de cuestiones relacionadas con aspectos personales: sexo, edad, formación, situación laboral y salidas profesionales.

La segunda parte se trataba de identificar las llamadas competencias genéricas, un total de 30, clasificadas en tres grupos: instrumentales (competencias que tienen una función instrumental), interpersonales (capacidades individuales relativas a la capacidad de expresar los propios sentimientos, habilidades críticas y de autocrítica) y las sistémicas (son las destrezas y habilidades que conciernen a los sistemas como totalidad).

Este bloque solicitaba dos tipos de respuesta, es decir, se les pidió a los encuestados que para cada una de las competencias genéricas respondiesen:

- La importancia de la destreza o competencia para el ejercicio de su profesión.

- El nivel de realización o logro en el ejercicio de la destreza o competencia que ellos consideraban que habían logrado como resultado de sus estudios universitarios.

Para la valoración de cada uno de estos aspectos se empleó una escala de 1 a 4 , y cuyo significado es el siguiente: 1 = nada; 2 = poco; 3 = bastante; 4 = mucho.

Además de indicar la importancia y el nivel de realización de cada una de las treinta competencias, se les pidió a los encuestados que eligieran y ordenaran las cinco competencias que consideraran más importantes, según su opinión.

La tercera parte del cuestionario esta referida a las competencias específicas de su titulación. Esta formada por un total de 17, que los alumnos debían clasificar por orden de importancia según su criterio.

El tratamiento de los datos se ha analizado a través del paquete estadístico SPSS (v. 11.0), realizándose un análisis descriptivo utilizando porcentajes y frecuencias. Los gráficos se han realizado a través del programa Excel 2000. 


\section{Resultados del estudio}

\section{Datos personales y profesionales}

La mayoría de los encuestados son mujeres (93\%), con una media de edad de 22 años (Gráfico 1), con estudios previos del campo de la educación, principalmente en educación infantil y primaria.

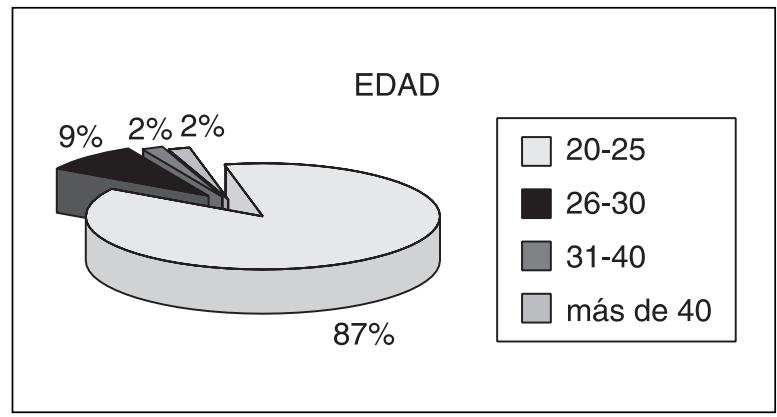

GRÁFICO 1.

Edad de los encuestados.

Los alumnos/as de la encuesta afirman que realizan la carrera de Psicopedagogía para ampliar sus estudios, aunque también realizan otro tipo de actividades relacionadas con este campo como: monitores de tiempo libre, clases particulares, cuidado de niños.

La formación recibida en la Universidad la consideran como normal, es decir, ni bastante buena ni tampoco mala, aunque las salidas profesionales de dicha carrera la valoran como pocas en general (Gráfico 2).

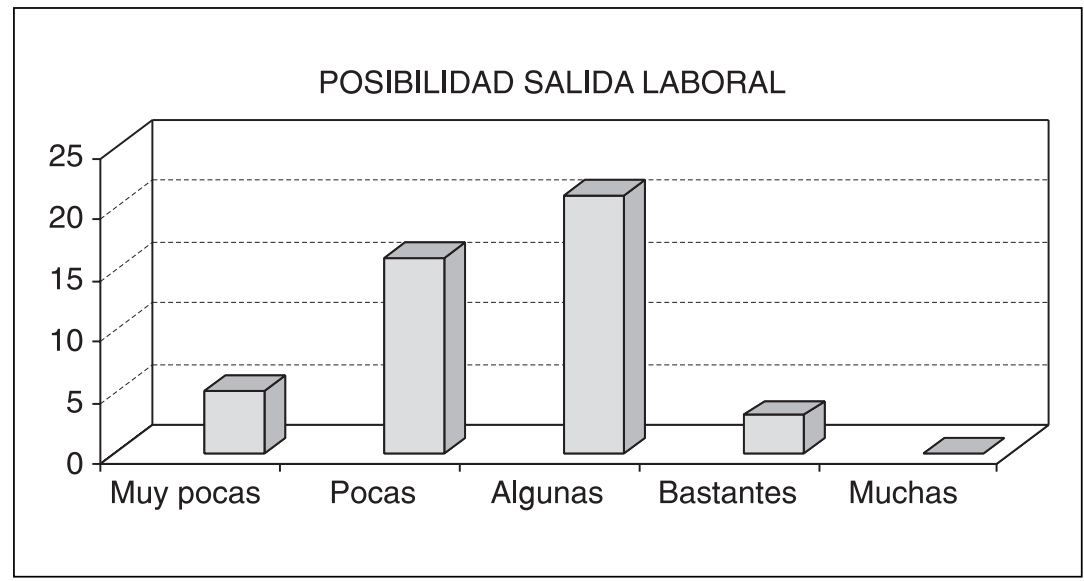

GRÁFICO 2.

Valoración de las posibles salidas laborales de la Licenciatura de Psicopedagogía. 


\section{Competencias genéricas dentro de la Licenciatura de Psicopedagogía}

Las competencias genéricas que los encuestados valoran como "muy" o "bastantes" importantes para su propio desarrollo profesional dentro del campo de la Psicopedagogía son Capacidad de aplicar los conocimientos en la práctica; Comunicación oral y escrita en la lengua; Capacidad de aprender; Habilidades de Investigación; Conocimiento de cultura y costumbres de otros países (Gráfico 3).

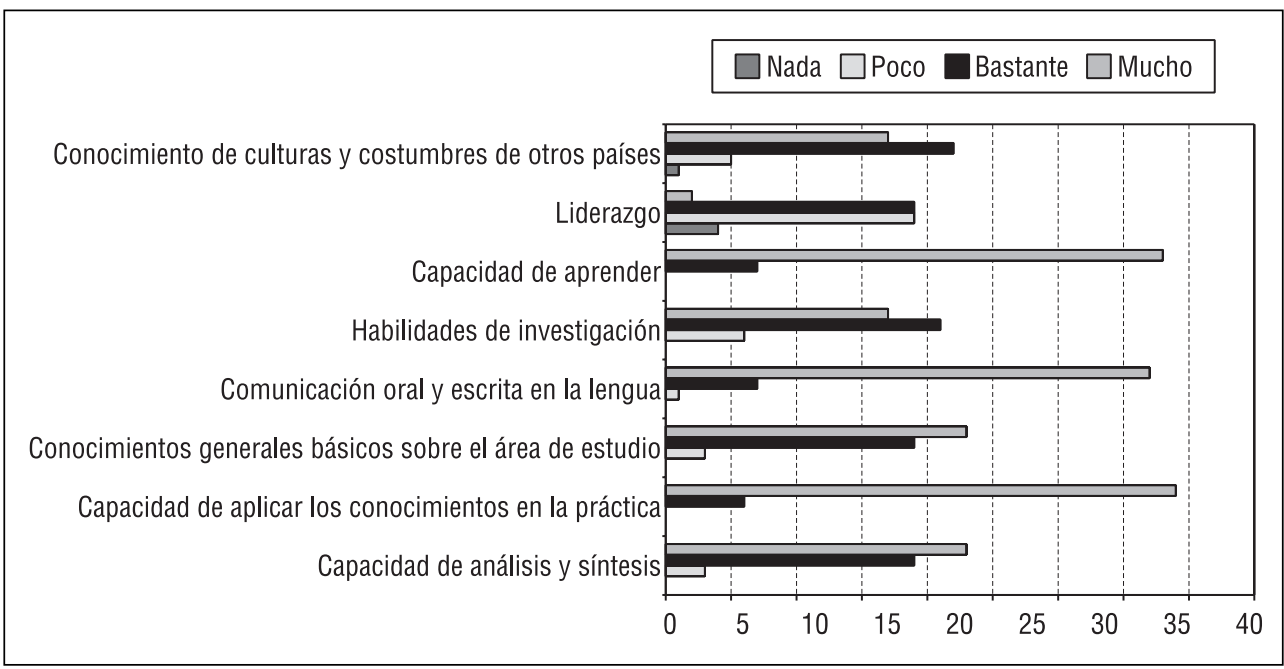

GRÁFICO 3.

Valoración de la importancia de las Competencias Genéricas dentro de la Licenciatura de Psicopedagogía.

\section{El nivel de desarrollo de las Competencias Genéricas en la Universidad}

Los alumnos encuestados afirman que las competencias que se han desarrollado con más profundidad en la Universidad han sido: Conocimientos generales básicos sobre el área de estudio; Conocimientos básicos de la profesión; Comunicación oral y escrita en la lengua; Capacidad crítica y autocrítica; trabajo en equipo y Apreciación de la diversidad y multiculturalidad (Gráfico 4).

Las Competencias que menos se han desarrollado en la Universidad son para los encuestados Resolución de problemas y Habilidad para trabajar en un contexto internacional.

En cuanto al apartado de la elección de las cinco competencias que consideran más importantes según la opinión de los alumnos de Psicopedagogía son las que aparecen en el gráfico 5 . 


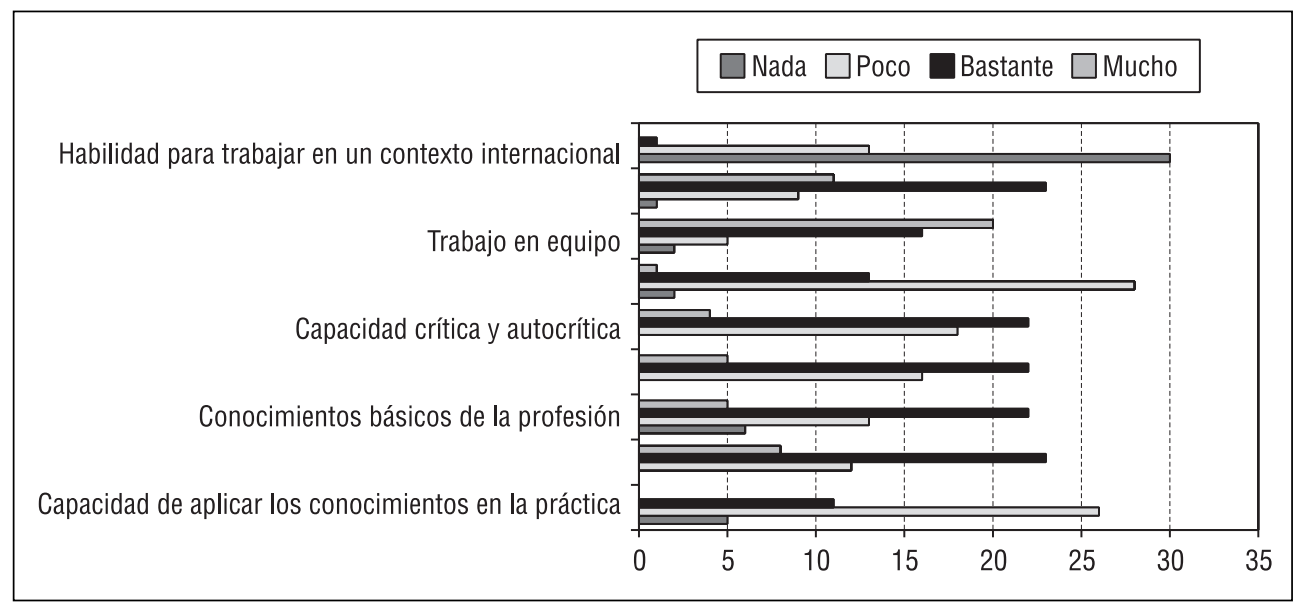

GRÁFICO 4.

Nivel de desarrollo de las Competencias Genéricas en la Universidad.

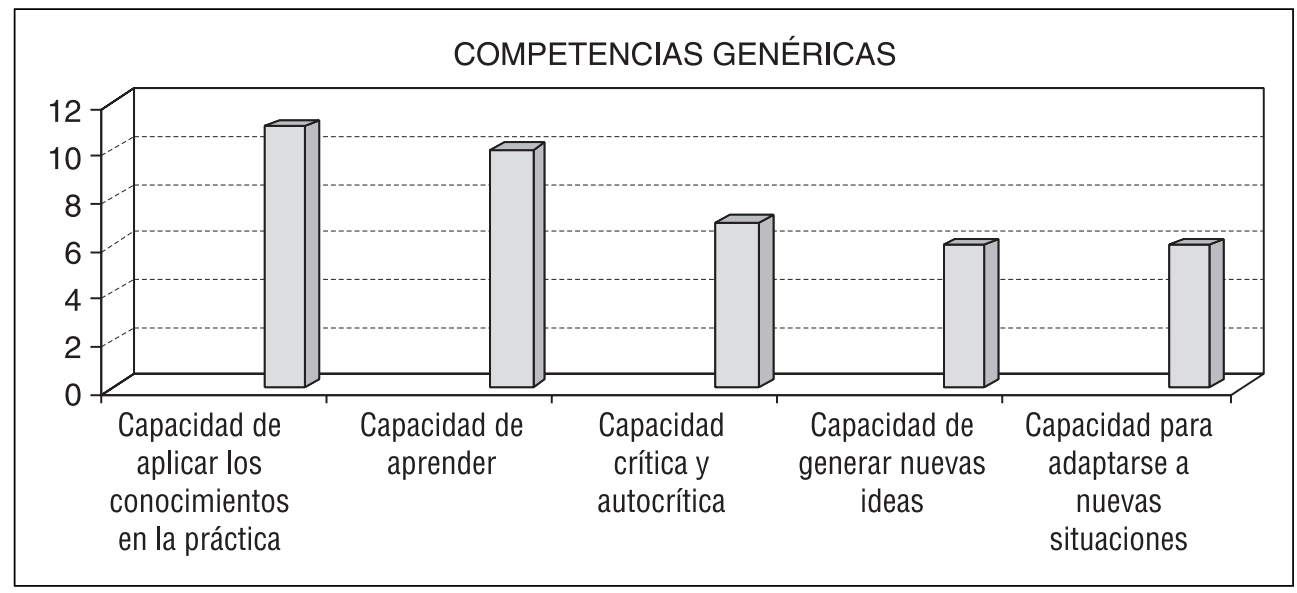

GRÁFICO 5.

Principales Competencias Genéricas.

\section{Competencias Específicas en los estudiantes de Psicopedagogía}

Como indicamos en el apartado anterior, para el análisis de estas Competencias, los encuestados debían valorar en una escala de 1 (más importante) a 17 (menos importante) una serie de competencias específicas.

Las Competencias Específicas más importantes para el desarrollo profesional de los estudiantes de Psicopedagogía son: Capacidad de aprender (32\%); Capacidad de aplicar los 
conocimientos a la práctica (14\%); Compromiso ético (12\%) y Conocimientos básicos de la profesión (12\%) (Gráfico 6).

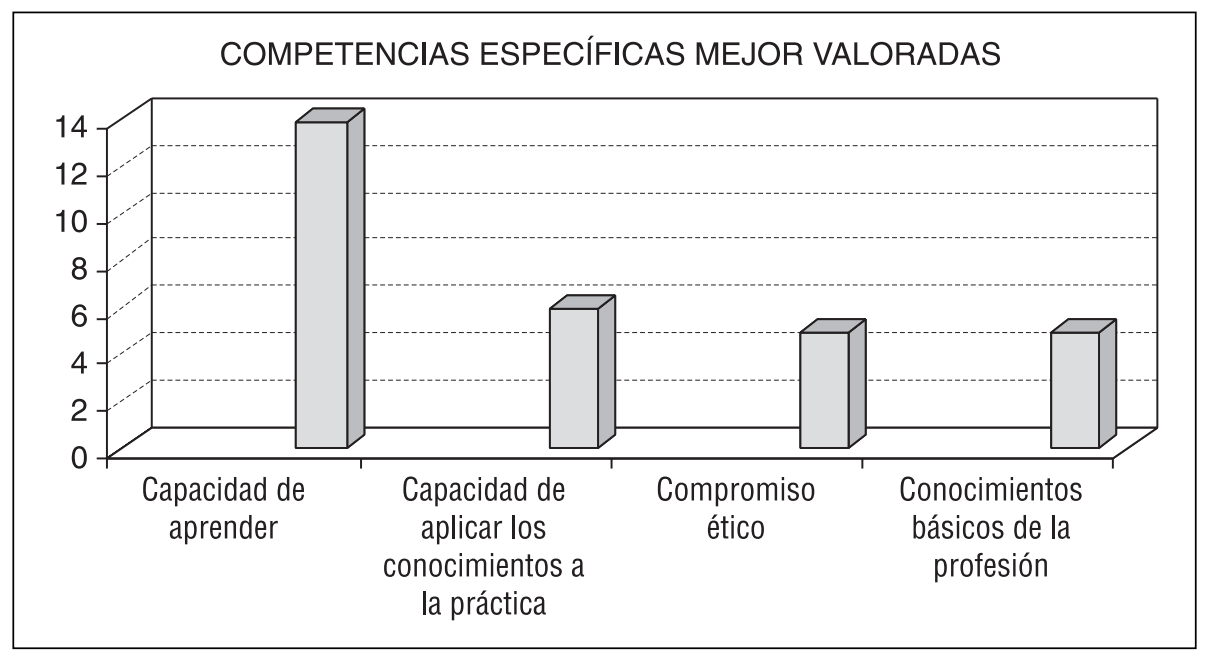

GRÁFICO 6.

Valoración de las Competencias Específicas más importantes para los estudiantes de Psicopedagogía.

En cambio, las competencias específicas menos valoradas son: Conocimiento de una segunda lengua (36\%); Habilidades básicas de una segunda lengua (18\%) y Habilidades de investigación (18\%).

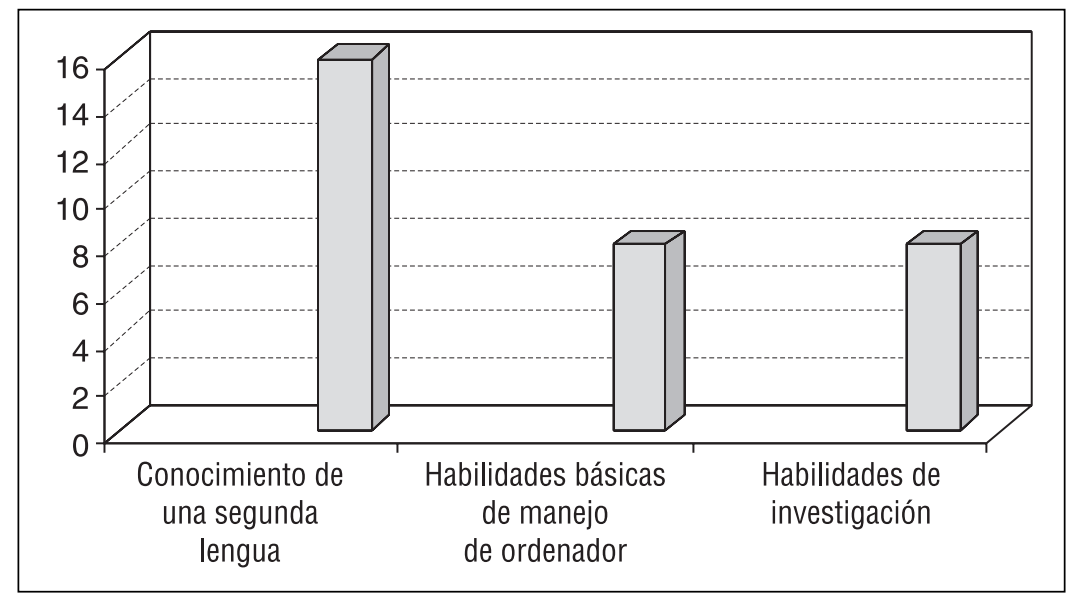

GRÁFICO 7.

Competencias Específicas menos valoradas por los estudiantes de Psicopedagogía. 


\section{Conclusiónes}

Lo más destacado de este estudio piloto podría estar relacionado con el poco interés al conocimiento de una segunda lengua, cuestión que les aproximaría, en cuanto titulados, hacia la Unión Europea.

Por otra parte se observa la baja expectativa de empleabilidad que le conceden a la titulación, esto es coincidente con el informe de la evaluación de la titulación del 2003. En este sentido de la empleabilidad hay que añadir que casi no contemplan yacimientos de empleo en las organizaciones públicas y en la empresa privada. En cambio se está constatando que, con cierta frecuencia nos encontramos a egresados psicopedagogos en los ámbitos de formación en la empresa y que, en muchas ocasiones, los empleadores requieren estos perfiles competenciales para trabajar en sus organizaciones.

\section{Referencias bibliográficas}

Blovin, M y Bergeron, C. (1997). Dictionaire de la readaption, t.2, temes d'intervention et d'aides tecniques. Québec.

Carneiro, R. (1999). Educación para la ciudadanía y las ciudades educadoras. Proyecto educativo de ciudad: la educación clave para el conocimiento y la convivencia Institutt d'educacio. Ajuntement de Barcelona, pp. 168-2003.

Coca, J. A. (2004). educaweb.com (consultado 7-X-2004).

Del Rincón, B. (2001). Presente y futuro del trabajo psicopedagógico. Barcelona. Ed. Ariel.

Echeverría, B. (2002). Gestión de la Competencia de Acción Profesional. Barcelona: Universidad de Barcelona.

Echeverría, B. (2004). Competencias profesionales, mercado laboral y educación superior. Conferencia Internacional de Orientación Inclusión social y desarrollo de la carrera. A Coruña. Libro de Actas. Ed. Tórculo.

Echeverría, B. (2005): Competencia de acción de los profesionales de la orientación. Madrid. Ed. Esic.

Echevarría, J. (1999). Los señores del aire: Telépolis y el tercer entorno. Barcelona. Ed. Destino.

Ferrer, F. (1998). Educación y sociedad en una nueva visión para el siglo XXI. Revista española de Educación Comparada. Madrid. UNED 11-35.

García-Fuentes de la Fuente, C. D. (2002). Proyecto Docente. (Inédito).

Pérez Escoda, N. (2001). Formación Ocupacional. Proyecto docente e investigador. Departamento de Métodos de Investigación y Diagnóstico en Educación. Barcelona: Universidad de Barcelona.

Repetto, R., Malik, B. y Ballesteros, B. (1998). Validación del cuestionario español de formación del personal que trabaja en orientación basada en competencias Revista de orientación y Psicopedagogía, Vol. $9, \mathrm{n}^{\circ} 15,1^{\circ}$ trimestre, pp. 56-86.

Repetto, R., Malik, B. y Ballesteros, B. (2003). Competences internationales pour les profesionnelles d'orientation educationelle et de carriére. En Report Final de International Conference, Quality development in vocational counselling and training. Bern. Suisse Ed. SVB-ASOSP.

Sanz, R. y Sobrado, L. (1998). Roles y funciones de los orientadores. Revista de Investigación Educativa, vol. $16, \mathrm{n}^{\circ} 2$, pp. 25-57. 


\section{ANEXO I}

\section{Cuestionario sobre competencias}

A continuación se presentan una serie de cuestiones que tienen que ver con las competencias y habilidades que pueden ser importantes para el buen desempeño de su profesión. Por favor, conteste a cada una de las preguntas. Las respuestas pueden ser de gran utilidad para la mejora de la planificación de su carrera de cara a los futuros alumnos. Rodee, en cada pregunta, la respuesta que considere más oportuna.

\section{Agradecemos sinceramente su colaboración}

1. Edad en años:

2. Sexo:

Hombre

Mujer

3. Año en que terminó sus estudios:

4. Nombre del título que obtuvo:

5. Situación laboral actual:

Trabajando en un puesto relacionado con sus estudios

Trabajando en un puesto no relacionado con sus estudios

Ampliando estudios

Buscando el primer empleo

En paro, habiendo trabajado antes

No estoy buscando ni he buscado empleo

Otro. Especificar, por favor:

6. ¿Cree que la formación que ha recibido en la universidad ha sido la adecuada?

$$
\text { Mucho } \square \quad \text { Bastante } \square \quad \text { Algo } \square \quad \text { Poco } \square \quad \text { Nada } \square
$$

7. ¿Cómo valora las posibles salidas profesionales de su titulación?

$$
\text { Mucho } \square \quad \text { Bastante } \square \quad \text { Algo } \square \quad \text { Poco } \square \quad \text { Nada }
$$

Para cada una de las competencias que se presentan a continuación, indique por favor:

- la importancia que, en su opinión, tiene la competencia o habilidad para el ejercicio de su profesional. 
- el nivel en que cree que la habilidad o competencia se ha desarrollado durante sus estudios en su universidad.

Puede utilizar los espacios en blanco para incluir alguna otra competencia que considere importante y que no aparece en el listado.

Utilice, por favor, la siguiente escala:

$1=$ nada; $2=$ poco 3 = bastante $4=$ mucho

\begin{tabular}{|c|c|c|}
\hline Habilidad/Competencia & Importancia & $\begin{array}{c}\text { Nivel en el } \\
\text { que se ha } \\
\text { desarrollado } \\
\text { en la } \\
\text { Universidad }\end{array}$ \\
\hline \multicolumn{3}{|l|}{ 1. Capacidad de análisis y síntesis } \\
\hline 2. Capacidad de aplicar los conocimientos en la práctica & 1234 & 1234 \\
\hline 3. Planificación y gestión del tiempo & 1234 & 1234 \\
\hline $\begin{array}{l}\text { 4. Conocimientos generales básicos sobre el área de } \\
\text { estudio }\end{array}$ & 1234 & 1234 \\
\hline 5. Conocimientos básicos de la profesión & 1234 & 1234 \\
\hline 6. Comunicación oral y escrita en la lengua & 1234 & 1234 \\
\hline 7. Conocimiento de una segunda lengua & 1234 & 1234 \\
\hline 8. Habilidades básicas de manejo del ordenador & 1234 & 1234 \\
\hline 9. Habilidades de investigación & 1234 & 1234 \\
\hline 10. Capacidad de aprender & 1234 & 1234 \\
\hline 11. Habilidades de gestión de la información & 1234 & 1234 \\
\hline 12. Capacidad crítica y autocrítica & 1234 & 1234 \\
\hline 13. Capacidad para adaptarse a nuevas situaciones & 1234 & 1234 \\
\hline 14. Capacidad para generar nuevas ideas (creatividad) & 1234 & 1234 \\
\hline 15. Resolución de problemas & 1234 & 1234 \\
\hline 16. Toma de decisiones & 1234 & 1234 \\
\hline 17. Trabajo en equipo & 1234 & 1234 \\
\hline 18. Habilidades interpersonales & 1234 & 1234 \\
\hline 19. Liderazgo & 1234 & 1234 \\
\hline 20. Capacidad de trabajar en un equipo multidisciplinar & 1234 & 1234 \\
\hline $\begin{array}{l}\text { 21. Capacidad para comunicarse con personas no expertas } \\
\text { en la materia }\end{array}$ & 1234 & 1234 \\
\hline 22. Apreciación de la diversidad y multiculturalidad & 1234 & 1234 \\
\hline 23. Habilidad para trabajar en un contexto internacional & 1234 & 1234 \\
\hline
\end{tabular}




\begin{tabular}{|c|c|c|}
\hline Habilidad/Competencia & Importancia & $\begin{array}{c}\text { Nivel en el } \\
\text { que se ha } \\
\text { desarrollado } \\
\text { en la } \\
\text { Universidad }\end{array}$ \\
\hline 24. Conocimiento de culturas y costumbres de otros países & 1234 & 1234 \\
\hline 25. Habilidad para trabajar de forma autónoma & 1234 & 1234 \\
\hline 26. Diseño y gestión de proyectos & 1234 & 1234 \\
\hline 27. Iniciativa y espíritu emprendedor & 1234 & 1234 \\
\hline 28. Compromiso ético & 1234 & 1234 \\
\hline 29. Preocupación por la calidad & 1234 & 1234 \\
\hline 30. Motivación de logro & 1234 & 1234 \\
\hline 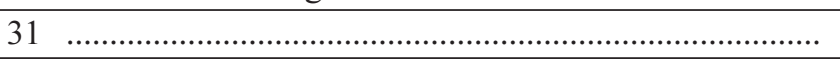 & 1234 & 1234 \\
\hline 32 & 1234 & 1234 \\
\hline 33 & 1234 & 1234 \\
\hline
\end{tabular}

Por favor a continuación elija y ordene las cinco competencias que considere más importantes según su opinión. Para ello escriba el número del ítem en los recuadros que aparecen abajo. Marque en la primera casilla la competencia que considera, en primer lugar, la más importante. En la segunda casilla señale la segunda competencia más importante, y así sucesivamente.

\section{1. Ítem número \\ 2. Ítem número \\ 3. Ítem número \\ 4. Ítem número \\ 5. Ítem número}

Aquí debajo se enumeran 17 competencias que han sido consideradas como las más importantes para el desarrolla profesional de los graduados universitarios, tanto por estos como por las empresas que los emplean.

Por favor, ordene estas 17 competencias en orden de importancia de acuerdo a su opinión. (1 es lo más importante y 17 lo menos).

Es vital que usted ordene TODAS y no brinde a una competencia un orden igual a otro. 


\begin{tabular}{|l|l|}
\hline \multicolumn{1}{|c|}{ Competencias genéricas } & Ranking \\
\hline 1. Capacidad de trabajar en un equipo interdisciplinar & \\
\hline 2. Apreciación de la diversidad y multiculturalidad & \\
\hline 3. Conocimientos generales básicos sobre el área de estudio & \\
\hline 4. Conocimientos básicos de la profesión & \\
\hline 5. Capacidad de análisis y síntesis & \\
\hline 6. Capacidad de aplicar los conocimientos en la práctica & \\
\hline 7. Capacidad para generar nuevas ideas (creatividad) & \\
\hline 8. Capacidad para adaptarse a nuevas situaciones & \\
\hline 9. Capacidad de aprender & \\
\hline 10. Capacidad de crítica y autocrítica & \\
\hline 11. Toma de decisiones & \\
\hline 12. Habilidades básicas de manejo del ordenador & \\
\hline 13. Compromiso ético & \\
\hline 14. Habilidades interpersonales & \\
\hline 15. Conocimiento de un segunda lengua & \\
\hline 16. Comunicación oral y escrita en la propia lengua & \\
\hline 17. Habilidades de investigación & \\
\hline
\end{tabular}

